Punjab University Journal of Mathematics (2021),53(11),765-777

https://doi.org/10.52280/pujm.2021.531101

\title{
New integral inequalities of Hermite-Hadamard type in a generalized context
}

Juan Eduardo Nápoles Valdés

UNNE, FaCENA, Ave. Libertad 5450, Corrientes 3400, Argentina

and

UTN-FRRE, French 414, Resistencia, Chaco 3500, Argentina

Email:jnapoles@exa.unne.edu.ar

Bahtiyar Bayraktar

Department of Educational Sciences

Uludag University, Bursa, Turkey

Email:bbayraktar@uludag.edu.tr

Saad Ihsan Butt

COMSATS University Islamabad

Lahore Campus, Pakistan

Email:saadihsanbutt@gmail.com

Received: 07 May, 2021 / Accepted: 23 October, 2021 / Published online: 25 November, 2021

\begin{abstract}
In this paper, we obtained new integral inequalities of the Hermite-Hadamard type for convex and quasi-convex functions in a generalized context.
\end{abstract}

\section{AMS (MOS) Subject Classification Codes:26D10, 26A51, 39B62, 26A33 \\ Key Words: Hermite-Hadamard integral inequality, weighted integral operators, quasi-convex functions.}

\section{INTRODUCTION}

To speak of convexity is to speak of interdisciplinary subjects. Convexity is a basic notion in geometry, but it is also widely used in other areas of mathematics. The use of convexity techniques appears in many branches of mathematics and science, such as the Theory of Optimization, Theory of Inequalities, Functional Analysis, Mathematical Programming, Theory of Games, Number Theory, Variational Calculus and their interrelationship with these branches have shown today deeper and more fruitful impact. In addition, in recent years, various extensions and generalizations of the classical concept of convexity of both sets and functions have been studied and there are regular meetings and conferences of researchers working in this area.

In our work, we will use the class of quasi-convex functions. 
Definition 1.1. A function $\psi: I \rightarrow \mathbb{R}, I:=\left[v_{1}, v_{2}\right]$ is said to be quasi-convex if $\psi(\theta x+(1-\theta) y) \leq \max \{\psi(x), \psi(y)\}$ holds for all $x, y \in I$ and $\theta \in[0,1]$.

It is known that a convex function is a quasi-convex function, but the converse is not always true, for example the floor function is quasi-convex but not convex, $\log (x)$ is concave and quasi-convex, and see an example in [9].

One of the most important inequalities, that has attracted many inequalities experts in the last few decades, is the Hermite-Hadamard inequality:

$$
\psi\left(\frac{v_{1}+v_{2}}{2}\right) \leq \frac{1}{v_{2}-v_{1}} \int_{v_{1}}^{v_{2}} \psi(\theta) d \theta \leq \frac{\psi\left(v_{1}\right)+\psi\left(v_{2}\right)}{2}
$$

holds for any function $\psi$ convex on the interval $\left[v_{1}, v_{2}\right]$.

Inequality ( 1.1 ) gives an estimate for the mean value of a function on a closed interval. This inequality was obtained by Charles Hermite in 1883 and Jacques Salomon Hadamard in 1893 independently of each other. The interested reader is referred to $[2-4,6,8,11-$ $14,16,18,20,21,24]$ and references therein for more information and other extensions of the Hermite-Hadamard inequality. All through the work we utilize the classical Gamma functions $\Gamma$ (see $[23,30,31]$ ) and $\Gamma_{k}$ (see [8]), where $\Gamma$ is the classic Gamma function and $\Gamma_{k}$ is called $k$-Gamma function:

$$
\Gamma(z)=\int_{0}^{\infty} \theta^{z-1} e^{-t} d \theta, \Re(z)>0 \text { and } \Gamma_{k}(z)=\int_{0}^{\infty} \theta^{z-1} e^{-\theta^{k} / k} d \theta, k>0 .
$$

Unmistakably, if $k \rightarrow 1$, we have $\Gamma_{k}(z) \rightarrow \Gamma(z), \Gamma_{k}(z)=(k)^{\frac{z}{k}-1} \Gamma\left(\frac{z}{k}\right)$ and $\Gamma_{k}(z+k)=$ $z \Gamma_{k}(z)$. To encourage comprehension of the subject, we present the definition of RiemannLiouville fractional integral (with $0 \leq v_{1}<\theta<v_{2} \leq \infty$ ). The first is the classic Riemann-Liouville fractional integrals.

Definition 1.2. The Riemann-Liouville fractional integrals $I_{v_{1}+}^{\alpha} \psi(x)$ and $I_{v_{2}-}^{\alpha} \psi(x)$ of order $\alpha \in \mathbb{C}, \Re(\alpha)>0$ are defined respectively by:

$$
\begin{aligned}
& \left.I_{v_{1}+}^{\alpha} \psi(x)=\frac{1}{\Gamma(\alpha)} \int_{v_{1}}^{x}(x-\theta)^{\alpha-1} \psi(\theta)\right) d \theta, \quad x>v_{1}, \\
& I_{v_{2}-}^{\alpha} \psi(x)=\frac{1}{\Gamma(\alpha)} \int_{x}^{v_{2}}(\theta-x)^{\alpha-1} \psi(\theta) d \theta, \quad x<v_{2},
\end{aligned}
$$

where $\psi \in L\left[v_{1}, v_{2}\right]$.

Remark 1.3. The Riemann-Liouville integral operator fulfills some essential properties of an operator of this nature, thus we have:

- $d_{\theta} I^{\alpha+1} \psi(\theta)=I^{\alpha} \psi(\theta)$, so $I^{\alpha}$ takes the role of an anti-derivative.

- $I^{\alpha}\left(I^{\beta} \psi(\theta)\right)=I^{\alpha+\beta} \psi(\theta)$, i. e., $I^{\alpha}$ satisfies the semigroup property. 
Definition 1.4. ( [19]) The $k$-Riemann-Liouville fractional integrals ${ }_{k} I_{v_{1}+}^{\alpha} \psi(x)$ and ${ }_{k} I_{v_{2}-}^{\alpha} \psi(x)$ of order $\alpha>0$ with $v_{1}>0$ are defined respectively by:

$$
\begin{aligned}
& { }_{k} I_{v_{1}+\psi}^{\alpha} \psi(x)=\frac{1}{k \Gamma_{k}(\alpha)} \int_{v_{1}}^{x}(x-\theta)^{\frac{\alpha}{k}-1} \psi(\theta) d \theta, \quad x>v_{1}, k>0 \\
& { }_{k} I_{v_{2}-\psi}^{\alpha} \psi(x)=\frac{1}{k \Gamma_{k}(\alpha)} \int_{x}^{v_{2}}(\theta-x)^{\frac{\alpha}{k}-1} \psi(\theta) d \theta, \quad x<v_{2}, k>0,
\end{aligned}
$$

where $\psi \in L\left[v_{1}, v_{2}\right]$.

Non-comformable fractional integral definitions ( [20]):

Definition 1.5. Let $\alpha \in \mathbb{R}$ and $0<v_{1}<v_{2}$. For each function $\psi \in L\left[v_{1}, v_{2}\right]$, we define

$$
N_{3} J_{u}^{\alpha} \psi(x)=\int_{u}^{x} \theta^{-\alpha} \psi(\theta) d \theta, \text { for every } x, u \in\left[v_{1}, v_{2}\right] \text {. }
$$

Definition 1.6. Let $\alpha \in \mathbb{R}$ and $v_{1}<v_{2}$. For each function, $\psi \in L\left[v_{1}, v_{2}\right]$. Let us define the Non-comformable fractional integrals

$$
\begin{aligned}
& N_{3} J_{v_{1}^{+}}^{\alpha} \psi(x)=\int_{v_{1}}^{x}(x-\theta)^{-\alpha} \psi(\theta) d \theta, \\
& N_{3} J_{v_{2}^{-}}^{\alpha} \psi(x)=\int_{x}^{v_{2}}(\theta-x)^{-\alpha} \psi(\theta) d \theta
\end{aligned}
$$

for every $x \in\left[v_{1}, v_{2}\right]$. Here, for $\alpha=0 \quad N_{N_{3}} J_{v_{1}^{+}}^{\alpha} \psi(x)={ }_{N_{3}} J_{v_{2}^{-}}^{0} \psi(x)=\int_{v_{1}}^{v_{2}} \psi(\theta) d \theta$.

In different works, integral operators have been used [1,7,27], which come from local differential operators of a different nature. Thus we have the Non-comformable fractional integrals $([15,20])$. Next, we present the weighted integral operators, which will provide a basis for our work.

Definition 1.7. Let $\psi \in L\left[v_{1}, v_{2}\right]$ and $w: I=[0,1] \rightarrow \mathbb{R}$, be a continuous and positive function, with first and second order derivatives piecewise continuous on I, taking in 0 and 1 we take the lateral derivatives, right and left respectively, and $w(0)=w(1)=0$. Then, the weighted integral operators are defined by (right and left, respectively):

$$
\begin{aligned}
& { }_{\Delta}^{w} I_{v_{1}^{+}} \psi(x)=\int_{v_{1}}^{x} w^{\prime \prime}\left(\frac{x-\theta}{\Delta}\right) \psi(\theta) d \theta, \quad x>v_{1}, \\
& { }_{\Delta}^{w} I_{v_{2}^{-}} \psi(x)=\int_{x}^{v_{2}} w^{\prime \prime}\left(\frac{\theta-x}{\Delta}\right) \psi(\theta) d \theta, \quad x<v_{2},
\end{aligned}
$$

where $\Delta=v_{2}-v_{1}$.

Remark 1.8. Given the generality of the kernel $w^{\prime \prime}(t)$ considered in the previous Definition, it may be that some of the integral operators obtained as particular cases of our definition do not satisfy some of the properties referred to the classical fractional operators, for example, the semi-group law. It is clear that in the cases that we indicate next in the Remark 1.9, if the referred properties are satisfied. 
Remark 1.9. Depending on the form of the function $w(\theta)$ from ( 1.2$)$, we get different integral operators:

1.If we take $w=w(\theta, \alpha)=\frac{\Delta^{\alpha-1} \theta^{\alpha+1}}{\alpha(\alpha+1) \Gamma(\alpha)}$, we get

Riemann-Liouville fractional integrals;

2.If we take $w=w(\theta, \alpha)=\frac{\Delta^{\frac{\alpha}{k}-1} \theta^{\frac{\alpha}{k}+1}}{\frac{\alpha}{k}\left(\frac{\alpha}{k}+1\right) k \Gamma_{k}(\alpha)}$, we get

$k$ - Riemann-Liouville fractional integrals;

3.If we take $w=w(\theta, \alpha)=\frac{\Delta^{-\alpha} \theta^{2-\alpha}}{(1-\alpha)(2-\alpha)}$, we get

Non-comformable fractional integrals;

4.If we take $w=w(\theta, 0)$, i.e. $w^{\prime \prime} \equiv 1$ we obtain the classical Riemann integrals

In this paper, we obtained new variants of the inequality (1.1) within the framework of the weigted integral operators of the Definition 1.7 for convex and quasi-convex functions.

\section{MAIN RESULTS}

Then, we can formulate our first result, which was used throughout the work.

Lemma 2.1. Let $\psi$ be a real function defined on some interval $I \subset \mathbb{R}$, twice differentiable on $I^{\circ}, v_{1}, v_{2} \in I^{\circ}, v_{1}<v_{2}$. If $\psi^{\prime \prime} \in L\left[v_{1}, v_{2}\right]$ and $w(0)=w(1)=0$, then the equality:

$$
\begin{aligned}
& \left(w^{\prime}(0) \psi\left(v_{2}\right)-w^{\prime}(1) \psi\left(v_{1}\right)\right)+\frac{1}{\Delta}\left[{ }_{\Delta}^{w} I_{v_{1}^{+}} \psi\left(v_{2}\right)\right] \\
& =\Delta^{2} \int_{0}^{1} w(\theta) \psi^{\prime \prime}\left(\theta v_{1}+(1-\theta) v_{2}\right) d \theta
\end{aligned}
$$

is valid.

Proof. Integrating by parts, we obtain

$$
\begin{aligned}
\int_{0}^{1} w(\theta) \psi^{\prime \prime}\left(\theta v_{1}+(1-\theta) v_{2}\right) d \theta & =\left\{\frac{1}{\Delta^{2}}\left[w^{\prime}(0) \psi\left(v_{2}\right)-w^{\prime}(1) \psi\left(v_{1}\right)\right]\right. \\
& \left.+\frac{1}{\Delta^{2}} \int_{0}^{1} w^{\prime \prime}(\theta) \psi\left(\theta v_{1}+(1-\theta) v_{2}\right) d \theta\right\} .
\end{aligned}
$$

By putting $z=\theta v_{1}+(1-\theta) v_{2}$, so $d z=\left(v_{1}-v_{2}\right) d \theta$; with this change of variables and rearranging the terms, we obtain equality ( 2.3 ).

Similarly, we can prove the validity of the next lemma:

Lemma 2.2. Let $\psi$ be a real function defined on some interval $I \subset \mathbb{R}$, twice differentiable on $I^{\circ}, v_{1}, v_{2} \in I^{\circ}, v_{1}<v_{2}$. If $\psi^{\prime \prime} \in L\left[v_{1}, v_{2}\right]$ and $w(0)=w(1)=0$, then the equality:

$$
\begin{aligned}
& \left(w^{\prime}(0) \psi\left(v_{1}\right)-w^{\prime}(1) \psi\left(v_{2}\right)\right)+\frac{1}{\Delta}\left[{ }_{\Delta}^{w} I_{v_{2}^{-}} \psi\left(v_{1}\right)\right] \\
& =\Delta^{2} \int_{0}^{1} w(\theta) \psi^{\prime \prime}\left((1-\theta) v_{1}+\theta v_{2}\right) d \theta
\end{aligned}
$$


is valid.

Remark 2.3. If we take $w(\theta)=1-(1-\theta)^{\alpha+1}-\theta^{\alpha+1}$, then from ( 2. 3 ), we get the result in [29] (see Lemma 4.1) and in [5] (see Lemma 2.2 ).

Remark 2.4. The above result contains as a particular case of Lemma 1 of [2] and Lemma 4 of [8] with $w(\theta)=\theta(1-\theta)$.

The first fundamental result of our work is the following. For simplicity let us denote

$$
\begin{aligned}
& w^{\prime}(0) \psi\left(v_{2}\right)-w^{\prime}(1) \psi\left(v_{1}\right)+\frac{1}{\Delta}\left[{ }_{\Delta}^{w} I_{v_{1}^{+}} \psi\left(v_{2}\right)\right]=L^{+}(H H) \\
& w^{\prime}(0) \psi\left(v_{1}\right)-w^{\prime}(1) \psi\left(v_{2}\right)+\frac{1}{\Delta}\left[{ }_{\Delta} I_{v_{2}^{-}} \psi\left(v_{1}\right)\right]=L^{-}(H H)
\end{aligned}
$$

Theorem 2.5. If, in addition to the conditions of Lemma 2.1, $\left|\psi^{\prime \prime}\right|$ is quasi-convex on $\left[v_{1}, v_{2}\right]$, then the inequality

$$
\left|L^{+}(H H)\right| \leq \Delta^{2} \cdot B \cdot \max \left\{\left|\psi^{\prime \prime}\left(v_{1}\right)\right|,\left|\psi^{\prime \prime}\left(v_{2}\right)\right|\right\}
$$

is valid, with $B=\int_{0}^{1} w(\theta) d \theta, \Delta=v_{2}-v_{1}$.

Proof. From the quasi-convexity of $\left|\psi^{\prime \prime}\right|$ and Lemma 2.1, we get

$$
\begin{aligned}
\left|L^{+}(H H)\right| & \leq \Delta^{2} \int_{0}^{1} w(\theta)\left|\psi^{\prime \prime}\left(\theta v_{1}+(1-\theta) v_{2}\right)\right| d \theta \\
& \leq \Delta^{2} \max \left\{\left|\psi^{\prime \prime}\left(v_{1}\right)\right|,\left|\psi^{\prime \prime}\left(v_{2}\right)\right|\right\} \int_{0}^{1} w(\theta) d \theta \\
& =\Delta^{2} \cdot B \cdot \max \left\{\left|\psi^{\prime \prime}\left(v_{1}\right)\right|,\left|\psi^{\prime \prime}\left(v_{2}\right)\right|\right\}
\end{aligned}
$$

Which is what was required to prove.

Remark 2.6. If we consider that $w(\theta)=\theta(1-\theta)$, then this result becomes Theorem 3 of [2].

From now on, we will use the well-known Hölder Inequality and its consequence, the socalled Power Mean Inequality, for more details we recommend the reader consult [10, 17]. In particular, we will use the following result.

Theorem 2.7. (Power-mean integral inequality). Let $q \geq 1$ and $1 / p+1 / q=1$. If $\psi$ and $\phi$ are real functions defined on $\left[v_{1}, v_{2}\right]$ and if $|\psi|,|\psi||\phi|$ are integrable functions on $\left[v_{1}, v_{2}\right]$ then

$$
\int_{v_{1}}^{v_{2}}|\psi(\theta) \phi(\theta)| d \theta \leq\left(\int_{v_{1}}^{v_{2}}|\psi(\theta)| d \theta\right)^{1-\frac{1}{q}}\left(\int_{v_{1}}^{v_{2}}|\psi(\theta)||\phi(\theta)|^{q} d \theta\right)^{\frac{1}{q}} .
$$

We can improve the previous result if we impose additional conditions to the quasiconvexity of $\left|\psi^{\prime \prime}\right|$. 
Theorem 2.8. Under assumptions of Lemma 2.1, if $\left|\psi^{\prime \prime}\right|^{q}$ is quasi-convex on $\left[v_{1}, v_{2}\right]$, for $q>1$, then the inequality

$$
\left|L^{+}(H H)\right| \leq B_{p} \cdot \Delta^{2} \cdot\left(\max \left\{\left|\psi^{\prime \prime}\left(v_{1}\right)\right|^{q},\left|\psi^{\prime \prime}\left(v_{2}\right)\right|^{q}\right\}\right)^{\frac{1}{q}}
$$

is valid, with $\frac{1}{p}+\frac{1}{q}=1, B_{p}=\left(\int_{0}^{1} w^{p}(\theta) d \theta\right)^{\frac{1}{p}}$ and $\Delta=v_{2}-v_{1}$.

Proof. From Hölder's inequality, in its integral version, and Lemma 2.1, we have

$$
\begin{aligned}
\left|L^{+}(H H)\right| & \leq \Delta^{2} \int_{0}^{1} w(\theta)\left|\psi^{\prime \prime}\left(\theta v_{1}+(1-\theta) v_{2}\right)\right| d \theta \\
& \leq \Delta^{2}\left(\int_{0}^{1} w(\theta)^{p} d \theta\right)^{\frac{1}{p}}\left(\int_{0}^{1}\left|\psi^{\prime \prime}\left(\theta v_{1}+(1-\theta) v_{2}\right)\right|^{q} d \theta\right)^{\frac{1}{q}} \\
& \leq \Delta^{2} \cdot B_{p} \cdot\left(\max \left\{\left|\psi^{\prime \prime}\left(v_{1}\right)\right|^{q},\left|\psi^{\prime \prime}\left(v_{2}\right)\right|^{q}\right\}\right)^{\frac{1}{q}}
\end{aligned}
$$

Which is what was required to prove.

Remark 2.9. Theorem 4 of [2] is easily obtained from the previous result if we put $w(\theta)=$ $\theta(1-\theta)$.

A more general variant of the previous theorem, is given in the following result.

Theorem 2.10. Under assumptions of Lemma 2.1, if $\left|\psi^{\prime \prime}\right|^{q}$ is quasi-convex on $\left[v_{1}, v_{2}\right]$ for $q \geq 1$, then the inequality

$$
\left|L^{+}(H H)\right| \leq B \cdot \Delta^{2} \cdot\left(\max \left\{\left|\psi^{\prime \prime}\left(v_{1}\right)\right|^{q},\left|\psi^{\prime \prime}\left(v_{2}\right)\right|^{q}\right\}\right)^{\frac{1}{q}}
$$

is valid, with $B$ and $\Delta$ is like in Theorem 2.5 .

Proof. Taking into account the of Lemma 2.1 and the power mean inequality (other form of Hölder's inequality) and $\frac{1}{p}+\frac{1}{q}=1$ for $q \geq 1$, we have

$$
\begin{aligned}
& \left|L^{+}(H H)\right| \leq \Delta^{2} \int_{0}^{1} w(\theta)\left|\psi^{\prime \prime}\left(\theta v_{1}+(1-\theta) v_{2}\right)\right| d \theta \\
& =\Delta^{2} \int_{0}^{1}[w(\theta)]^{\frac{1}{p}+\frac{1}{q}}\left|\psi^{\prime \prime}\left(\theta v_{1}+(1-\theta) v_{2}\right)\right| d \theta \\
& \leq \Delta^{2}\left(\int_{0}^{1} w(\theta) d \theta\right)^{1-\frac{1}{q}}\left(\int_{0}^{1} w(\theta)\left|\psi^{\prime \prime}\left(\theta v_{1}+(1-\theta) v_{2}\right)\right|^{q} d \theta\right)^{\frac{1}{q}} \\
& =\Delta^{2} \cdot B \cdot\left(\max \left\{\left|\psi^{\prime \prime}\left(v_{1}\right)\right|^{q},\left|\psi^{\prime \prime}\left(v_{2}\right)\right|^{q}\right\}\right)^{\frac{1}{q}} .
\end{aligned}
$$

Which is what was required to prove.

Remark 2.11. We can verify, without much difficulty, that Theorem 5 of [2] is a particular case of the previous result if we make $w(\theta)=\theta(1-\theta)$.

The following theorem is obvious. 
Theorem 2.12. If, in addition to the conditions of Lemma 2.1, $\left|\psi^{\prime \prime}\right|$ is convex on $\left[v_{1}, v_{2}\right]$, then the inequality

$$
\left|L^{+}(H H)+L^{-}(H H)\right| \leq \Delta^{2} \cdot B \cdot\left(\left|\psi^{\prime \prime}\left(v_{1}\right)\right|+\left|\psi^{\prime \prime}\left(v_{2}\right)\right|\right)
$$

is valid, with $B=\int_{0}^{1} w(\theta) d \theta$ and $\Delta=v_{2}-v_{1}$.

Proof. By taking into account equalities ( 2. 3 ), ( 2. 4 ) and the properties of the modulus from the condition of convexity of the function $\left|\psi^{\prime \prime}\right|$, we get:

$$
\begin{aligned}
& \left|L^{+}(H H)+L^{-}(H H)\right|=\Delta^{2} \mid \int_{0}^{1} w(\theta) \psi^{\prime \prime}\left(\theta v_{1}+(1-\theta) v_{2}\right) d \theta \\
& \quad+\int_{0}^{1} w(\theta) \psi^{\prime \prime}\left((1-\theta) v_{1}+\theta v_{2}\right) d \theta \mid \\
& \leq \Delta^{2} \int_{0}^{1} w(\theta)\left|\psi^{\prime \prime}\left(\theta v_{1}+(1-\theta) v_{2}\right)+\psi^{\prime \prime}\left((1-\theta) v_{1}+\theta v_{2}\right)\right| d \theta \\
& \leq \Delta^{2} \int_{0}^{1} w(\theta)\left|\theta \psi^{\prime \prime}\left(v_{1}\right)+(1-\theta) \psi^{\prime \prime}\left(v_{2}\right)+(1-\theta) \psi^{\prime \prime}\left(v_{1}\right)+\theta \psi^{\prime \prime}\left(v_{2}\right)\right| d \theta \\
& =\Delta^{2} \int_{0}^{1} w(\theta)\left|\psi^{\prime \prime}\left(v_{1}\right)+\psi^{\prime \prime}\left(v_{2}\right)\right| d \theta \\
& \leq \Delta^{2}\left(\left|\psi^{\prime \prime}\left(v_{1}\right)\right|+\left|\psi^{\prime \prime}\left(v_{2}\right)\right|\right) \int_{0}^{1} w(\theta) d \theta=\Delta^{2} \cdot B \cdot\left(\left|\psi^{\prime \prime}\left(v_{1}\right)\right|+\left|\psi^{\prime \prime}\left(v_{2}\right)\right|\right) .
\end{aligned}
$$

The proof is completed.

Remark 2.13. We can verify, without much difficulty, that Theorem 3.1 ( for $s=1$ and $m=1$ ) of [3] is a special case of the Theorem 2.12 if we make $w(\theta)=\theta^{\alpha}(1-\theta)$.

\section{SOME METHODOLOGICAL OBSERVATIONS}

Throughout the work, we have pointed out the generality of our results since they contain as particular cases, several of which are known from the literature. However, we can reformulate our Definition 1.7 and cover other known results. For example, it suffices to use a version of our integral operator:

Definition 3.1. Let $\psi \in L\left[v_{1}, v_{2}\right]$ and let $w$ be a continuous and positive function, $w$ : $I=[0,1] \rightarrow \mathbb{R}$, with the first and second order derivatives piecewise continuous on $I$ and $w(0)=0$. Then, the weighted integral operators are defined by (right and left side, respectively):

$$
\begin{aligned}
{ }_{\frac{\Delta}{2}}^{w} I_{v_{1}^{+}} \psi(x) & =\int_{v_{1}}^{x} w^{\prime \prime}\left(\frac{x-\theta}{\frac{\Delta}{2}}\right) \psi(\theta) d \theta, \quad x>v_{1}, \\
{ }_{\frac{\Delta}{2}}^{w} I_{v_{2}^{-}} \psi(x) & =\int_{x}^{v_{2}} w^{\prime \prime}\left(\frac{\theta-x}{\frac{\Delta}{2}}\right) \psi(\theta) d \theta, \quad x<v_{2}, \\
\text { where } \Delta & =v_{2}-v_{1} .
\end{aligned}
$$

So, we have 
Lemma 3.2. Let $\psi$ be a function of real variables defined on the interval $I, I \subset \mathbb{R}$, twice differentiable on $I^{\circ}, v_{1}, v_{2} \in I^{\circ}, v_{1}<v_{2}$ and $w(0)=0$. If $\psi^{\prime \prime} \in L\left[v_{1}, v_{2}\right]$, then we have the following equality:

$$
\begin{aligned}
& {\left[w^{\prime}(0) \frac{\psi\left(v_{1}\right)+\psi\left(v_{2}\right)}{2}-w^{\prime}(1) \psi\left(\frac{v_{1}+v_{2}}{2}\right)\right]} \\
& \quad+\frac{1}{\Delta}\left[\frac{\Delta}{2} I_{\frac{v_{1}+v_{2}}{2}}+\psi\left(v_{2}\right)+\frac{w}{2} I_{\frac{v_{1}+v_{2}}{2}}-\psi\left(v_{1}\right)\right] \\
& =\frac{\Delta^{2}}{8} \int_{0}^{1} w(\theta)\left[\psi^{\prime \prime}\left(\frac{\theta}{2} v_{1}+\frac{2-\theta}{2} v_{2}\right)+\psi^{\prime \prime}\left(\frac{2-\theta}{2} v_{1}+\frac{\theta}{2} v_{2}\right)\right] d \theta
\end{aligned}
$$

where $\Delta=v_{2}-v_{1}$.

Proof. Analogous to the proof of Lemma 2.1 in [18]. By integrating by parts and changing variables under the resulting integrals, we obtain:

$$
\begin{aligned}
& \int_{0}^{1} w(\theta) \psi^{\prime \prime}\left(\frac{\theta}{2} v_{1}+\frac{2-\theta}{2} v_{2}\right) d \theta \\
& =-\frac{2 w(1)}{\Delta} \psi^{\prime}\left(\frac{v_{1}+v_{2}}{2}\right)-\frac{4 w^{\prime}(1)}{\Delta^{2}} \psi\left(\frac{v_{1}+v_{2}}{2}\right)+\frac{4 w^{\prime}(0)}{\Delta^{2}} \frac{\psi\left(v_{1}\right)+\psi\left(v_{2}\right)}{2} \\
& \quad+\frac{8}{\Delta^{3}} \int_{\frac{v_{1}+v_{2}}{2}}^{v_{2}} w^{\prime \prime}\left(\frac{v_{2}-z}{\frac{\Delta}{2}}\right) \psi(z) d z \\
& =-\frac{2 w(1)}{\Delta} \psi^{\prime}\left(\frac{v_{1}+v_{2}}{2}\right)-\frac{4 w^{\prime}(1)}{\Delta^{2}} \psi\left(\frac{v_{1}+v_{2}}{2}\right)+\frac{4 w^{\prime}(0)}{\Delta^{2}} \frac{\psi\left(v_{1}\right)+\psi\left(v_{2}\right)}{2} \\
& \quad+\frac{8}{\Delta^{3}}\left[\frac{w}{\frac{\Delta}{2}} I_{\frac{v_{1}+v_{2}}{2}}+\psi\left(v_{2}\right)\right] .
\end{aligned}
$$

Similarly, for the second integral, we will have

$$
\begin{aligned}
& \int_{0}^{1} w(\theta) \psi^{\prime \prime}\left(\frac{2-\theta}{2} v_{1}+\frac{\theta}{2} v_{2}\right) d \theta=\frac{2 w(1)}{\Delta} \psi^{\prime}\left(\frac{v_{1}+v_{2}}{2}\right) \\
& -\frac{4 w^{\prime}(1)}{\Delta^{2}} \psi\left(\frac{v_{1}+v_{2}}{2}\right)+\frac{4 w^{\prime}(0)}{\Delta^{2}} \frac{\psi\left(v_{1}\right)+\psi\left(v_{2}\right)}{2}+\frac{8}{\Delta^{3}}\left[\frac{\Delta}{2} I_{\frac{v_{1}+v_{2}}{2}}-\psi\left(v_{1}\right)\right] .
\end{aligned}
$$

By adding ( 3.10) and (3.11) and multiplying by $\frac{\left(v_{2}-v_{1}\right)^{2}}{8}$ we get (3.9). The proof is completed.

Remark 3.3. In a particular case, if we take $w(\theta)=\theta^{\alpha+1}$, then from (3.9), we get the result in [28] (see Lemma 1 ).

Remark 3.4. In a particular case, if we take $w(\theta)=\theta^{2}$, then from ( 3.9 ), we get the result in [26] (see Lemma 2 ).

By using Lemma 3.2, one can easily prove the following theorem: 
Theorem 3.5. Under assumptions of Lemma 3.2, if $\left|\psi^{\prime \prime}\right|$ is convex on $\left[v_{1}, v_{2}\right]$, we have the following inequality

$$
\begin{aligned}
& \left|A+\frac{1}{\Delta}\left[\frac{w}{\frac{\Delta}{2}} I_{\frac{v_{1}+v_{2}}{2}}+\psi\left(v_{2}\right)+{ }_{\frac{\Delta}{2}}^{w} I_{\frac{v_{1}+v_{2}}{2}}-\psi\left(v_{1}\right)\right]\right| \\
& \leq \frac{\Delta^{2}}{8} \cdot B \cdot\left(\left|\psi^{\prime \prime}\left(v_{1}\right)\right|+\left|\psi^{\prime \prime}\left(v_{2}\right)\right|\right),
\end{aligned}
$$

with $B=\int_{0}^{1} w(\theta) d \theta$ and $A=\left[w^{\prime}(0)\left(\frac{\psi\left(v_{1}\right)+\psi\left(v_{2}\right)}{2}\right)-w^{\prime}(1) \psi\left(\frac{v_{1}+v_{2}}{2}\right)\right]$.

Proof. Analogous to the proof of Theorem 2.1 in [18]. From Lemma 3.2 and the properties of the module, we will have:

$$
\begin{aligned}
& \mid w^{\prime}(0) \frac{\psi\left(v_{1}\right)+\psi\left(v_{2}\right)}{2}-w^{\prime}(1) \psi\left(\frac{v_{1}+v_{2}}{2}\right) \\
& \quad+\frac{1}{\Delta}\left[\frac{w}{2} I_{\frac{v_{1}+v_{2}}{2}}+\psi\left(v_{2}\right)+\frac{w}{2} I_{\frac{v_{1}+v_{2}}{2}}-\psi\left(v_{1}\right)\right] \mid \\
& \leq \frac{\Delta^{2}}{8} \int_{0}^{1} w(\theta)\left[\left|\psi^{\prime \prime}\left(\frac{\theta}{2} v_{1}+\frac{2-\theta}{2} v_{2}\right)\right|+\left|\psi^{\prime \prime}\left(\frac{2-\theta}{2} v_{1}+\frac{\theta}{2} v_{2}\right)\right|\right] d \theta
\end{aligned}
$$

From the condition of convexity of the function $\left|\psi^{\prime \prime}\right|$ :

$$
\begin{aligned}
& \leq\left|\psi^{\prime \prime}\left(\frac{\theta}{2} v_{1}+\frac{2-\theta}{2} v_{2}\right)\right|+\left|\psi^{\prime \prime}\left(\frac{2-\theta}{2} v_{1}+\frac{\theta}{2} v_{2}\right)\right| \\
& \leq \frac{\theta}{2}\left|\psi^{\prime \prime}\left(v_{1}\right)\right|+\left(1-\frac{\theta}{2}\right)\left|\psi^{\prime \prime}\left(v_{2}\right)\right|+\left(1-\frac{\theta}{2}\right)\left|\psi^{\prime \prime}\left(v_{1}\right)\right|+\frac{\theta}{2}\left|\psi^{\prime \prime}\left(v_{2}\right)\right| \\
& =\left|\psi^{\prime \prime}\left(v_{1}\right)\right|+\left|\psi^{\prime \prime}\left(v_{2}\right)\right|
\end{aligned}
$$

From ( 3.13 ), by taking into account ( 3.14 ) and the accepted designations, we obtain ( 3. 12 ). The proof is completed.

Remark 3.6. If we take $w(\theta)=\theta^{\alpha+1}$, then we obtain a particular case of Theorem 3.5:

$$
\begin{aligned}
& \mid \frac{2^{\alpha-1} \Gamma(\alpha+2)}{\Delta^{\alpha}}\left[I_{\frac{v_{1}+v_{2}}{2}}^{\alpha} \psi\left(v_{2}\right)+I_{\frac{v_{1}+v_{2}}{2}}^{\alpha} \psi\left(v_{1}\right)\right] \\
& -(\alpha+1) \psi\left(\frac{v_{1}+v_{2}}{2}\right) \mid \leq \frac{\Delta^{2}}{8(\alpha+2)}\left(\left|\psi^{\prime \prime}\left(v_{1}\right)\right|+\left|\psi^{\prime \prime}\left(v_{2}\right)\right|\right) .
\end{aligned}
$$

This inequality was obtained in [28] (see Theorem 3).

Remark 3.7. If we put $w(\theta)=\theta^{\frac{\alpha}{k}+1}$, then from Theorem 3.5, we obtain the inequality via $k$-fractional integral:

$$
\begin{aligned}
& \left|\frac{2^{\frac{\alpha}{k}-1} \alpha \Gamma_{k}(\alpha)}{\Delta^{\frac{\alpha}{k}}\left(\frac{\alpha}{k}+1\right)}\left[{ }_{k} I_{\frac{v_{1}+v_{2}}{2}}^{\alpha}+\psi\left(v_{2}\right)+{ }_{k} I_{\frac{v_{1}+v_{2}}{2}}^{\alpha}-\psi\left(v_{1}\right)\right]-\psi\left(\frac{v_{1}+v_{2}}{2}\right)\right| \\
& \leq \frac{\Delta^{2}}{8\left(\frac{\alpha}{k}+1\right)\left(\frac{\alpha}{k}+2\right)}\left(\left|\psi^{\prime \prime}\left(v_{1}\right)\right|+\left|\psi^{\prime \prime}\left(v_{2}\right)\right|\right) .
\end{aligned}
$$

This inequality was obtained in [18] (see Corollary 2.1). 
Remark 3.8. If we put $w(\theta)=\theta^{2-\alpha}$, then from Theorem 3.5, we obtain the inequality via non-conformable fractional integral:

$$
\begin{aligned}
& \left|\frac{(1-\alpha) \Delta^{\alpha-1}}{2^{\alpha}}\left[N_{3} I_{\frac{v_{1}+v_{2}}{2}}^{\alpha} \psi\left(v_{2}\right)+N_{3} I_{\frac{v_{1}+v_{2}}{2}}^{\alpha} \psi\left(v_{1}\right)\right]-\psi\left(\frac{v_{1}+v_{2}}{2}\right)\right| \\
& \leq \frac{\Delta^{2}}{8(1-\alpha)(2-\alpha)}\left(\left|\psi^{\prime \prime}\left(v_{1}\right)\right|+\left|\psi^{\prime \prime}\left(v_{2}\right)\right|\right) .
\end{aligned}
$$

Remark 3.9. If we put $w(\theta)=\theta^{2}$, then we obtain a particular case of Theorem 3.5:

$$
\left|\frac{1}{v_{2}-v_{1}} \int_{0}^{1} \psi(\theta) d \theta-\psi\left(\frac{v_{2}+v_{1}}{2}\right)\right| \leq \frac{\left(v_{2}-v_{1}\right)^{2}}{48}\left(\left|\psi^{\prime \prime}\left(v_{1}\right)\right|+\left|\psi^{\prime \prime}\left(v_{2}\right)\right|\right) .
$$

This estimate was obtained in [25] and confirmed in a number of works (for example [3], [4] and [22]).

Theorem 3.10. Under assumptions of Lemma 3.2, if $\left|\psi^{\prime \prime}\right|^{q}$ is convex on $\left[v_{1}, v_{2}\right]$, we have the following inequality:

$$
\begin{aligned}
& \left|A+\frac{1}{\Delta}\left[\frac{w}{\frac{\Delta}{2}} I_{\frac{v_{1}+v_{2}}{2}}+\psi\left(v_{2}\right)+\frac{w}{\frac{\Delta}{2}} I_{\frac{v_{1}+v_{2}}{2}}-\psi\left(v_{1}\right)\right]\right| \\
& \leq \frac{\Delta^{2}}{8} \cdot B_{p} \cdot\left[\left(\frac{\left|\psi^{\prime \prime}\left(v_{1}\right)\right|^{q}}{4}+\frac{3\left|\psi^{\prime \prime}\left(v_{2}\right)\right|^{q}}{4}\right)^{\frac{1}{q}}+\left(\frac{3\left|\psi^{\prime \prime}\left(v_{1}\right)\right|^{q}}{4}+\frac{\left|\psi^{\prime \prime}\left(v_{2}\right)\right|^{q}}{4}\right)^{\frac{1}{q}}\right],
\end{aligned}
$$

whith $A=\left[w^{\prime}(0)\left(\frac{\psi\left(v_{1}\right)+\psi\left(v_{2}\right)}{2}\right)-w^{\prime}(1) \psi\left(\frac{v_{1}+v_{2}}{2}\right)\right], \frac{1}{p}+\frac{1}{q}=1, \forall q, p>1$ and $B_{p}=$ $\left(\int_{0}^{1} w^{p}(\theta) d \theta\right)^{\frac{1}{p}}$.

Proof. Analogous to the proof of Theorem 2.2 in [18]. If we use the triangle inequality to the right-hand side of ( 3.9 ), we obtain:

$$
\begin{aligned}
& \mid\left[w^{\prime}(0) \frac{\psi\left(v_{1}\right)+\psi\left(v_{2}\right)}{2}-w^{\prime}(1) \psi\left(\frac{v_{1}+v_{2}}{2}\right)\right] \\
& \quad+\frac{1}{\Delta}\left[\frac{w}{2} I_{\frac{v_{1}+v_{2}}{2}}+\psi\left(v_{2}\right)+\frac{w}{\frac{\Delta}{2}} I_{\frac{v_{1}+v_{2}}{2}}-\psi\left(v_{1}\right)\right] \mid \\
& \leq \frac{\Delta^{2}}{8} \int_{0}^{1} w(\theta)\left[\left|\psi^{\prime \prime}\left(\frac{\theta}{2} v_{1}+\frac{2-\theta}{2} v_{2}\right)\right|+\left|\psi^{\prime \prime}\left(\frac{2-\theta}{2} v_{1}+\frac{\theta}{2} v_{2}\right)\right|\right] d \theta .
\end{aligned}
$$


By using the well-known Hölder integral inequality and since $\left|\psi^{\prime \prime}\right|^{q}$ is a convex function for the right-side ( 3.15 ), we get:

$$
\begin{aligned}
& \frac{\Delta^{2}}{8} \int_{0}^{1} w(\theta)\left[\left|\psi^{\prime \prime}\left(\frac{\theta}{2} v_{1}+\frac{2-\theta}{2} v_{2}\right)\right|+\left|\psi^{\prime \prime}\left(\frac{2-\theta}{2} v_{1}+\frac{\theta}{2} v_{2}\right)\right|\right] d \theta \\
& \leq \frac{\Delta^{2}}{8}\left(\int_{0}^{1} w^{p}(\theta) d \theta\right)^{\frac{1}{p}}\left[\left(\left|\psi^{\prime \prime}\left(v_{1}\right)\right|^{q} \int_{0}^{1} \frac{\theta}{2} d \theta+\left|\psi^{\prime \prime}\left(v_{2}\right)\right|^{q} \int_{0}^{1} \frac{2-\theta}{2} d \theta\right)^{\frac{1}{q}}\right. \\
& \left.\quad+\left(\left|\psi^{\prime \prime}\left(v_{1}\right)\right|^{q} \int_{0}^{1} \frac{2-\theta}{2} d \theta+\left|\psi^{\prime \prime}\left(v_{1}\right)\right|^{q} \int_{0}^{1} \frac{\theta}{2} d \theta\right)^{\frac{1}{q}}\right] \\
& =\frac{\Delta^{2} B_{p}}{8}\left[\left(\frac{\left|\psi^{\prime \prime}\left(v_{1}\right)\right|^{q}}{4}+\frac{3\left|\psi^{\prime \prime}\left(v_{2}\right)\right|^{q}}{4}\right)^{\frac{1}{q}}+\left(\frac{3\left|\psi^{\prime \prime}\left(v_{1}\right)\right|^{q}}{4}+\frac{\left|\psi^{\prime \prime}\left(v_{2}\right)\right|^{q}}{4}\right)^{\frac{1}{q}}\right] .
\end{aligned}
$$

By taking into account ( 3.15 ), the proof is completed.

Remark 3.11. It is not difficult to verify that the inequality

$$
\begin{aligned}
& \left|\frac{2^{\alpha-1} \Gamma(\alpha+2)}{\Delta^{\alpha}}\left[I_{\frac{v_{1}+v_{2}}{2}}^{\alpha} \psi\left(v_{2}\right)+I_{\frac{v_{1}+v_{2}}{2}}^{\alpha} \psi\left(v_{1}\right)\right]-(\alpha+1) \psi\left(\frac{v_{1}+v_{2}}{2}\right)\right| \\
& \leq \frac{\Delta^{2}}{8[(\alpha+1) p+1]^{\frac{1}{p}}} \\
& \times\left[\left(\frac{\left|\psi^{\prime \prime}\left(v_{1}\right)\right|^{q}}{4}+\frac{3\left|\psi^{\prime \prime}\left(v_{2}\right)\right|^{q}}{4}\right)^{\frac{1}{q}}+\left(\frac{3\left|\psi^{\prime \prime}\left(v_{1}\right)\right|^{q}}{4}+\frac{\left|\psi^{\prime \prime}\left(v_{2}\right)\right|^{q}}{4}\right)^{\frac{1}{q}}\right] .
\end{aligned}
$$

from [28] (Theorem 4) is a special case of Theorem 3.10 if we put $w(\theta)=\theta^{\alpha+1}$.

\section{CONCLusions}

In this work, we have obtained some inequalities by using a certain "weighted" integral, which contains several already published results and leaves open new lines of research as we pointed out in the previous section. Throughout the work, we have obtained the Hermite-Hadamard inequalities for the functions, whose second derivatives are convex and quasi-convex, via generalized integrals. To achieve our objectives, we obtained two lemmas, and on this basis, we obtained different types of integral identities for twice differentiable convex and quasi-convex functions, which were the generalizations of the classic Hermite-Hadamard Inequality.

Apart what we previously presented, other formulations of our results can be obtained in two directions: first, by imposing additional conditions on the function $w(\theta)$ and, secondly, by the other notions of convexity. With respect to the firstly indicated direction, we would like to make a final Remark, on the application of the results obtained for special means. Consider the means as arbitrary real numbers $v_{1}$ and $v_{2},\left(v_{1} \neq v_{2}\right)$. Be $A$, the arithmetic media and $L$, the generalized $\log -$ mean

$$
A(a, b)=\frac{a+b}{2} \text { and } L_{n}(a, b)=\left[\frac{b^{n+1}-a^{n+1}}{(n+1)(b-a)}\right]^{\frac{1}{n}}
$$


then, using the Theorem 2.5 for $w(\theta)=\theta(1-\theta)$, we obtain the following result.

Proposition 4.1. Let $v_{1}, v_{2} \in \mathbb{R}, v_{1}<v_{2}$ and $\psi(\theta)=\theta^{n}, t \in\left[v_{1}, v_{2}\right], n \in \mathbb{N}, n \geq 2$. Then, the following inequality holds

$$
\left|A\left(v_{1}^{n}, v_{2}^{n}\right)-L_{n}^{n}\left(v_{1}, v_{2}\right)\right| \leq \frac{\left(v_{2}-v_{1}\right)^{2} n(n-1)}{12} \max \left\{\left|v_{1}\right|^{n-2},\left|v_{2}\right|^{n-2}\right\} .
$$

Proof. From ( 2. 6 ), by taking into account ( 2.5 ), we get:

$$
\begin{aligned}
& \left|L^{+}(H H)\right| \leq\left(v_{2}-v_{1}\right)^{2} \cdot B \cdot \max \left\{\left|\psi^{\prime \prime}\left(v_{1}\right)\right|,\left|\psi^{\prime \prime}\left(v_{2}\right)\right|\right\} \\
& \left|L^{+}(H H)\right|=\left|w^{\prime}(0) \psi\left(v_{2}\right)-w^{\prime}(1) \psi\left(v_{1}\right)+\frac{1}{\Delta}\left[{ }_{\Delta}{ }_{\Delta} I_{1}^{+} \psi\left(v_{2}\right)\right]\right| \\
& =\left|v_{2}^{n}+v_{1}^{n}+\frac{1}{v_{2}-v_{1}} \int_{0}^{1}(-2) \theta^{n} d \theta\right|=2\left|\frac{v_{2}^{n}+v_{1}^{n}}{2}-\frac{v_{2}^{n+1}-v_{1}^{n+1}}{\left(v_{2}-v_{1}\right)(n+1)}\right| \\
& =2\left|A\left(v_{1}^{n}, v_{2}^{n}\right)-L_{n}^{n}\left(v_{1}, v_{2}\right)\right| .
\end{aligned}
$$

On the other hand, since $\psi^{\prime \prime}(\theta)=n(n-1) \theta^{n-2}$, we have:

$$
\begin{aligned}
& \left(v_{2}-v_{1}\right)^{2} \cdot B \cdot \max \left\{\left|\psi^{\prime \prime}\left(v_{1}\right)\right|,\left|\psi^{\prime \prime}\left(v_{2}\right)\right|\right\} \\
& =\left(v_{2}-v_{1}\right)^{2}\left(\int_{0}^{1} \theta(1-\theta) d \theta\right) n(n-1) \max \left\{\left|v_{1}\right|^{n-2},\left|v_{2}\right|^{n-2}\right\} \\
& =\frac{\left(v_{2}-v_{1}\right)^{2}}{6} n(n-1) \max \left\{\left|v_{1}\right|^{n-2},\left|v_{2}\right|^{n-2}\right\} .
\end{aligned}
$$

From ( 4.17), by taking into account (4.18) and (4.19), we obtain ( 4. 16 ). The proof is completed.

Remark 4.2. The obtained score is in line with the score presented in Proposition 1 of [2]. A similar observation is valid for Propositions 2 and 3 of the mentioned work.

\section{REFERENCES}

[1] A. O. Akdemir, S. I. Butt, M. Nadeem, M. A. Ragusa, New general variants of chebyshev type inequalities via generalized fractional integral operators, Mathematics., 9, No.2 (2021).

[2] M. Alomari, M. Darus, S. S. Dragomir, New inequalities of Hermite-Hadamard type for functions whose second derivatives absolute values are quasi-convex, RGMIA Res. Rep. Coll., 12, Supplement, Article 17 (2009).

[3] B. Bayraktar, Some integral inequalities of Hermite-Hadamard type for differentiable $(s, m)-$ convex functions via fractional integrals, TWMS Journal of Applied and Engineering Mathematics, 10, No. 3 (2020) 625-637.

[4] B. Bayraktar, Some new generalizations of Hadamard-Type Midpoint inequalities involving fractional integrals, Problemy Analiza - Issues of Analysis 9, No. 27-3 (2020) 66-82

[5] K. Boukerrioua, T. Chiheb, B. Meftah, Fractional Hermite-Hadamard type inequalities for functions whose second derivative are $(s, r)$-convex in the second sense, Kragujevac Journal of Mathematics 40, No. 2 (2016) 172-191.

[6] S. I. Butt, S. Yousaf, A. O. Akdemir, M. A Dokuyucu, New Hadamard-type integral inequalities via a general form of fractional integral operators, Chaos, Solitons \& Fractals, 148111025 (2021).

[7] S. I. Butt, A. O. Akdemir, J. Nasir, F. Jarad, Some Hermite-Jensen-Mercer Like Inequalities For Convex Functions Through A Certain Generalized Fractional Integrals And Related Results, Miskolc Mathematical Notes., 21,No.2 (2020) 689-715. 
[8] S. S. Dragomir, C. E. M. Pearce, Selected Topics on Hermite-Hadamard Inequalities, RGMIA Monographs, Victoria University (2000) ia aviable http://rgmia.vu.edu.au/monographs/hermite_hadamard.html

[9] D.A. Ion, Some estimates on the Hermite-Hadamard inequality through quasi-convex functions, Annals of the University of Craiova - Mathematics and Computer Science Series, 34 (2007) 82-87

[10] I. Iscan, New refinements for integral and sum forms of Hölder inequality, Journal of Inequalities and Applications 2019 (2019) 304

[11] M.A. Khan,Y. Khurshid, T. Ali, N. Rehman, Inequalities for Three Times Differentiable Functions, Punjab Uni. j. math. 49, No 2(2016) 35-48.

[12] M.A. Khan, Y. Khurshid, S.S. Dragomir, R. Ullah, New Hermite-Hadamard Type Inequalities With Applications, Punjab Uni. j. math. 50, No 3 (2018) 1-12.

[13] M.A. Latif, S.S Dragomir, S. Obeidat, New Inequalities of Fejer and Hermite-Hadamard type Concerning Convex and Quasi-Convex Functions With Applications, Punjab Uni. j. math. 53, No 2(2021) 1-17.

[14] M. A. Latif, S. S. Dragomir, E. Momoniat, Some Weighted Hermite-Hadamard-Noor Type Inequalities for Differentiable Preinvex and Quasi Preinvex Functions, Punjab Uni. j. math. 47, No 1(2015) 57-72.

[15] F. Martínez, P. O. Mohammed and J. E. Nápoles Valdés, Non Conformable Fractional Laplace Tranform, Kragujevac journal of mathematical, 46(2022), No. 3, 341-354.

[16] B. Meftah, Fractional Hermite-Hadamard Type Integral Inequalities for Functions whose Modulus of Derivatives are Co-ordinated log-Preinvex, Punjab Uni. j. math. 51, 2, 21-37, 2019.

[17] D. S. Mitrinovic, J. E. Pecaric, A. M. Fink, Classical and new inequalities in analysis, Kluwer Akademic Publishers, Dordrecht, Boston, London, 1993.

[18] P. O. Mohammed, M. Z. Sarikaya, On generalized fractional integral inequalities for twice dierentiable convex functions, Journal of Computational and Applied Mathematics 372 (2020) 112740

[19] S. Mubeen, G.M. Habibullah, $k$-Fractional integrals and application, International Journal of Contemporary Mathematical Sciences, 7, No. 2 (2012) 89-94.

[20] J. E. Nápoles V., J. M. Rodríguez, J.M. Sigarreta, New Hermite-Hadamard type inequalities involving Nonconformable integral operators, Symmetry 11, No 9:1108 (2019)

[21] J. E. Nápoles V., F. Rabossi, AD Samaniego, CONVEX FUNCTIONS: ARIADNE'S THREAD OR CHARLOTTE'S SPIDERWEB?, Advanced Mathematical Models and Applications 5, No. 2, (2020) 176-191

[22] S. Qaisar, F. Ahmad, S. S. Dragomir, M. Iqbal, New Hermite-Hadamard inequalities via fractional integrals, whose absolute values of second derivatives is P-convex, Journal of Mathematical Inequalities, 12, No. 3 (2018) 655-664.

[23] E.D. Rainville, Special Functions. Macmillan Co., New York (1960)

[24] S. Rashid, M.A. Noor, K.I. Noor, Integral Inequalities for Exponentially Geometrically Convex Functions via Fractional Operators,Punjab Uni. j. math. 52, No 6 (2020) 65-82.

[25] M. Z. Sarkkaya, N. Aktan, On the generalization of some integral inequalities and their applications, Mathematical and computer Modelling 54, N0 9 (2011) 2175-2182.

[26] M. Z. Sarıkaya, M. E. Kiris, Some new inequalities of Hermite-Hadamard type for s-convex functions, Miskolc Mathematical Notes,16, No 1 (2015) 491-501.

[27] E. Set, S. I. Butt, A. O. Akdemir, A. Karaoglan, T. Abdeljawad,New integral inequalities for differentiable convex functions via Atangana-Baleanu fractional integral operators, Chaos, Solitons \& Fractals., 143 (2021) 110554.

[28] M. Tomar, E. Set, M. Z. Sarıkaya, Hermite-Hadamard type Riemann-Liouville fractional integral inequalities for convex functions, AIP Conference Proceedings 1726 (2016) 020035.

[29] J. Wang, J. Deng, M. Feckan, Hermite-Hadamard-type inequalities for $r$-convex functions based on the use of Riemann-Liouville fractional integrals, Ukrainian mathematical journal 65, No 2 (2013) 193-211

[30] Yang, Z.-H., Tian, J.-F., Monotonicity and inequalities for the gamma function, J. Inequal. Appl. 2017(2017) 317.

[31] Yang, Z.-H., Tian, J.-F., Monotonicity and sharp inequalities related to gamma function, Journal of Mathematical Inequalities 12, No 1 (2018) 1-22. 